\title{
Dynamical analysis of a 2-degrees of freedom spatial pendulum
}

\author{
Stelian Alaci ${ }^{1, *}$, Florina-Carmen Ciornei $^{1}$, Sorinel-Toderas Siretean $^{1}$, Mariana-Catalina Ciornei $^{2}$ \\ and Gabriel Andrei Ţibu ${ }^{1}$ \\ ${ }^{1}$ Stefan cel Mare University, Suceava, 13 University Str., 720229, Romania \\ ${ }^{2}$ Carol Davila University of Medicine and Pharmacy, Dpt. Clinic 2, 8 Bd. Eroii Sanitari, 050474, Bucharest, Romania
}

\begin{abstract}
A spatial pendulum with the vertical immobile axis and horizontal mobile axis is studied and the differential equations of motion are obtained applying the method of Lagrange equations. The equations of motion were obtained for the general case; the only simplifying hypothesis consists in neglecting the principal moments of inertia about the axes normal to the oscillation axes. The system of nonlinear differential equations was numerically integrated. The correctness of the obtained solutions was corroborated to the dynamical simulation of the motion via dynamical analysis software. The perfect concordance between the two solutions proves the rightness of the equations obtained.
\end{abstract}

\section{Introduction}

Oscillatory motions occur frequently in natural world [1]. In mechanical engineering, they are met as vibrations when the elastic elements are associated with elements characterized by inertial properties (mass and moment of inertia). Another class of oscillatory motions are the oscillations themselves performed by bodies with one or several degrees of freedom actuated by a field (gravity, electrostatic etc.). The pendulum with one degree of freedom was in the attention of the scientist from old times [2]. An important application is the pendulum watch that allowed for precise time measurement. The attention for the oscillatory motions of the pendula has not lost the importance even in nowadays. One of the modern theories concerning the evolution of dynamical systems is related to the integration of differential equations that describe their behaviour. Thus, for the integration of a differential equation of an actual case, the initial conditions must be stipulated. The attempt of modelling more complex phenomena lead to the conclusion that it is possible that a minor variation of initial conditions may produce significant changes in the evolution of the system [3]. This property of a system to essentially modify its evolution for the smallest variations of initial conditions is known as chaos and the mathematical domain that studies the phenomenon is named the chaos theory [4].

\section{Obtaining the equations of motion of the 2 DOF spatial pendulum}

Looking for simple models where the chaos occurrence is possible it was recognized that the plane double pendulum is one of the simplest dynamical systems where chaos may happen. A plane pendulum with two degrees of freedom is made of two joined rigid bodies, one of the bodies has an additional immobile joint and the two axes are parallel. For studying the dynamical behaviour of the system, the equations of motion must be firstly established. The equations of motion have generally a complex form. Particular cases are considered for obtaining analytical solutions, though. Many of the scientific references consider the double mathematical pendulum or, at most, the double physical pendulum made of two identical homogenous rods, as in Fig. 1.
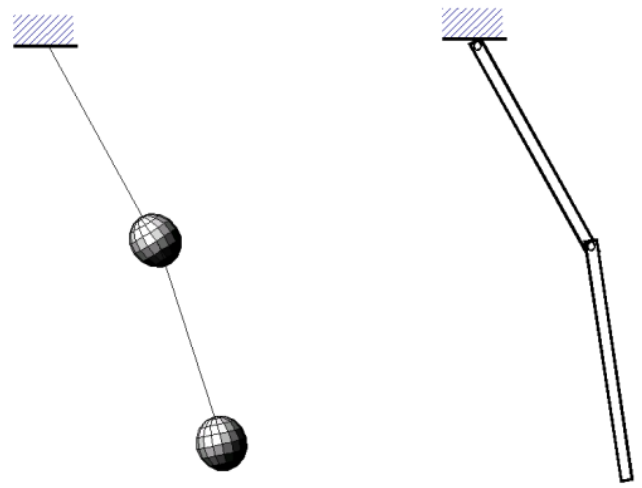

Fig. 1. Particular types of plane pendula with two DOF

The paper presents the analysis of the dynamical behaviour of a spatial pendulum with two DOF. The spatial pendulum with two degrees of freedom is presented in Fig. 2. It consists in two homogenous rods with negligible cross section, joined via a rotation pair. One of the rigid bodies is also jointed to the ground using a joint with vertical axis. Three coordinate systems are considered for the study of the motion: an immobile system $O x_{0} y_{0} z_{0}$ and two Cartesian mobile frames with the origins $O_{1}$ and $O_{2}$ positioned in the centers of the

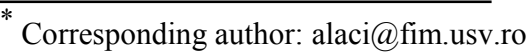


immobile and mobile joint, respectively. The axes of the rotation pairs are the $O_{1} z_{1}$ and $O_{2} z_{2}$ axes. The axes $O_{1} x_{1}$ and $\mathrm{O}_{2} x_{2}$ will pass through the centers of mass $G_{1}$ and $G_{2}$ of the two rigid bodies. The $O_{1} y_{1}, O_{2} y_{2}$ axes complete the coordinate systems in such a manner that they are right oriented. There are known: the masses of the bodies $M_{1}$ and $M_{2}$; the central moments of inertia $J_{G_{I}}$ and $J_{G_{2}}$ with respect to axes parallel to $O_{1} z_{1}$ and $O_{2} z_{2}$; the positions of the centers of mass précised by the distances $\xi_{1}$ and $\xi_{2}$. The external force acting upon the pendulum are the weights of the two rigid bodies, $m_{1} g$ and $m_{2} \boldsymbol{g}$. The position of the pendulum is completely specified by the angles $\theta$ and $\varphi$ that characterize the motions from the two rotation pairs.

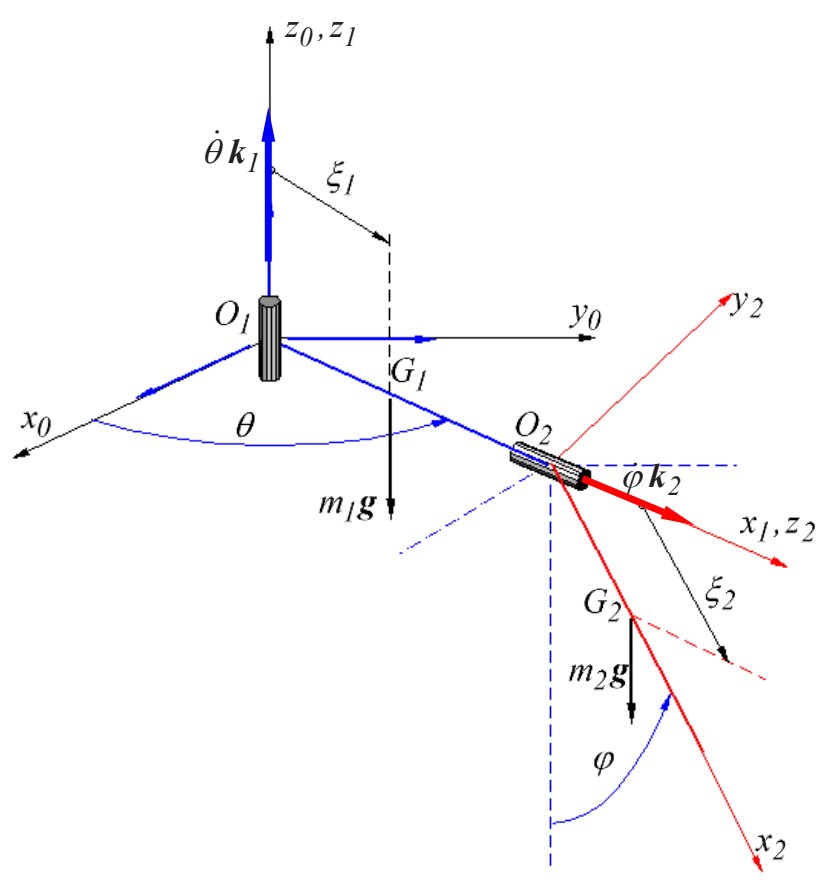

Fig. 2. Spatial pendulum with two DOF

The motion of the pendulum is characterized using the method of Lagrange equations [5] under the assumption that the friction is negligible in the two rotation pairs:

$$
\frac{d}{d t} \frac{\partial E}{\partial \dot{q}_{k}}-\frac{\partial E}{\partial q_{k}}=\sum_{i} \boldsymbol{F}_{i} \cdot \frac{\partial \boldsymbol{r}_{i}}{\partial q_{k}}
$$

where $E$ is the kinetical energy of the system, $q_{k}$ are generalized coordinates and $\boldsymbol{F}_{i}$ are the external forces. The next relation is applied for the calculus of kinetical energy:

$$
E=\frac{1}{2} \omega_{1}^{T} \boldsymbol{J}_{G_{1}} \omega_{1}+\frac{1}{2} M_{1} v_{G_{1}}^{2}+\frac{1}{2} \omega_{2}^{T} \boldsymbol{J}_{G_{2}} \omega_{2}+\frac{1}{2} M_{2} v_{G_{1}}^{2}
$$

In order to find the velocities of the centers of mass of the two elements, the position vectors of the centers of mass

\footnotetext{
* Corresponding author: alaci@fim.usv.ro
}

$\boldsymbol{r}_{G_{I}}$ and $\boldsymbol{r}_{G_{2}}$ are required. For the rigid linked to the ground:

$$
\boldsymbol{r}_{G_{I}}=\left[\begin{array}{lll}
\xi_{1} \cos \theta & \xi_{1} \sin \theta & 0
\end{array}\right]^{T}
$$

and the velocity of the center of mass $G_{1}$ is:

$$
v_{G_{1}}=\dot{\theta}\left[\begin{array}{lll}
-\xi_{1} \sin \theta & \xi_{1} \cos \theta & 0
\end{array}\right]
$$

The angular velocity of the rod joined to the ground is:

$$
\omega_{1}=\dot{\theta}\left[\begin{array}{lll}
0 & 0 & 1
\end{array}\right]^{T}
$$

The inertia matrixes of the rods with respect to the principal central axes have the general form:

$$
\boldsymbol{J}_{G_{1,2}}=\left[\begin{array}{ccc}
J_{1,2 x} & 0 & 0 \\
0 & J_{1,2 y} & 0 \\
0 & 0 & J_{1.2_{z}}
\end{array}\right]
$$

Assuming that the dimensions of the cross-section of the rods are negligible compared to their lengths, the following approximation is justified:

$$
J_{x_{1,2}}=J_{y_{1,2}} \cong 0
$$

The coordinate transformation relation [6-7] from the system 2 to the ground system is applied for finding the position vector of the center of mass $G_{2}$ in the ground frame:

$$
\boldsymbol{r}_{G_{2}}=\boldsymbol{Z}(\theta, 0) \boldsymbol{Y}\left(\frac{\pi}{2}, 0\right) \boldsymbol{Z}\left(\varphi, L_{1}\right)\left[\begin{array}{llll}
\xi_{2} & 0 & 0 & 1
\end{array}\right]^{T}
$$

Where, according to [7]:

$$
\boldsymbol{Z}(\gamma, c)=\left[\begin{array}{cccc}
\cos \gamma & -\sin \gamma & 0 & 0 \\
\sin \gamma & \cos \gamma & 0 & 0 \\
0 & 0 & 1 & c \\
0 & 0 & 0 & 1
\end{array}\right]
$$

and

$$
\boldsymbol{Y}(\beta, b)=\left[\begin{array}{cccc}
\cos \beta & 0 & \sin \beta & 0 \\
0 & 1 & 0 & b \\
-\sin \beta & 0 & \cos \beta & 0 \\
0 & 0 & 0 & 1
\end{array}\right]
$$

are the roto-translation operators about the axes $\mathrm{Oz}$ and $O y$ respectively and $L_{l}$ is the length of the first rod: 


$$
r_{G_{2}}=\left[\begin{array}{c}
-\xi_{2} \sin \theta \sin \varphi+L_{1} \cos \theta \\
\xi_{2} \cos \theta \sin \varphi+L_{1} \sin \theta \\
-\xi_{2} \cos \varphi
\end{array}\right]
$$

The derivative with respect to time of the relation 11 provides the velocity:

$$
\boldsymbol{v}_{G_{2}}=\left[\begin{array}{c}
-\xi_{2} \dot{\theta} \cos \theta \sin \varphi-\xi_{2} \dot{\varphi} \sin \theta \cos \varphi-L_{1} \dot{\theta} \sin \theta \\
-\xi_{2} \dot{\theta} \sin \theta \sin \varphi+\xi_{2} \dot{\varphi} \cos \theta \cos \varphi+L_{1} \dot{\theta} \cos \theta \\
\xi_{2} \dot{\varphi} \sin \varphi
\end{array}\right]
$$

The angular velocity of the rod with respect to the system in which the matrix $\boldsymbol{J}_{G_{2}}$ is presented is required for calculating the rotation kinetical energy of the element 2 . The angular velocity of the bar 2 is:

$$
\omega_{2}=\dot{\theta} \boldsymbol{k}_{1}+\dot{\varphi} \boldsymbol{k}_{2}=\left[\begin{array}{c}
-\dot{\theta} \cos \varphi \\
\dot{\theta} \sin \varphi \\
\dot{\varphi}
\end{array}\right]
$$

and it is employed in finding the kinetical energy of the pendulum:

$$
\begin{aligned}
& E_{c}=\frac{1}{2}\left[J_{O_{1}}+M_{2}\left(L_{1}^{2}+\xi_{2}^{2} \sin ^{2} \varphi\right)\right] \dot{\theta}^{2} \\
& +\frac{1}{2} J_{O_{2}} \dot{\varphi}^{2}+M_{2} \xi_{2} \dot{\theta} \dot{\varphi} L_{1} \cos \varphi \\
& \left\{\begin{array}{l}
\left(M_{2} \xi_{2}^{2} \sin ^{2} \varphi+J_{O_{1}}+M_{2} L_{1}^{2}\right) \ddot{\theta}+M_{2} L_{1} \xi_{2} \ddot{\varphi} \cos \varphi-M_{2} L_{1} \xi_{2} \dot{\varphi}^{2} \sin \varphi+M_{2} \xi_{2}^{2} \dot{\theta} \dot{\varphi} \sin 2 \varphi=0 \\
M_{2} L_{1} \xi_{2} \ddot{\theta} \cos \varphi+J_{O_{2}} \ddot{\varphi}-M_{2} \xi_{2}^{2} \dot{\theta}^{2} \sin \varphi \cos \varphi+M_{2} \xi_{2} g \sin \varphi=0
\end{array}\right. \\
& \left\{\begin{array}{l}
\ddot{\theta}=\left|\begin{array}{cc}
-M_{2} \xi_{2} L_{1} \dot{\varphi}^{2} \sin \varphi+M_{2} \xi_{2}^{2} \dot{\varphi} \dot{\theta} \sin (2 \varphi) & M_{2} L_{1} \xi_{2} \cos \varphi \\
-M_{2} \xi_{2}^{2} \dot{\theta}^{2} \sin \varphi \cos \varphi+M_{2} \xi_{2} g \sin \varphi & J_{O_{2}}
\end{array}\right| /\left|\begin{array}{cc}
J_{O_{1}}+M_{2} L_{1}^{2}+M_{2} \xi_{2}^{2} \sin ^{2} \varphi & M_{2} L_{1} \xi_{2} \cos \varphi \\
M_{2} L_{1} \xi_{2} \cos \varphi & J_{O_{2}}
\end{array}\right| \\
\ddot{\varphi}=\left|\begin{array}{cc}
J_{O_{1}}+M_{2} L_{1}^{2}+M_{2} \xi_{2}^{2} \sin ^{2} \varphi & -M_{2} L_{1} \xi_{2} \dot{\varphi}^{2}+M_{2} \xi_{2}^{2} \dot{\dot{\varphi}} \dot{\sin } 2 \varphi \\
M_{2} L_{1} \xi_{2} \cos \varphi & -M_{2} \xi_{2}^{2} \dot{\theta}^{2} \sin \varphi \cos \varphi+M_{2} \xi_{2} g \sin \varphi
\end{array}\right| /\left|\begin{array}{cc}
J_{O_{1}}+M_{2} L_{1}^{2}+M_{2} \xi_{2}^{2} \sin ^{2} \varphi & M_{2} L_{1} \xi_{2} \cos \varphi \\
M_{2} L_{1} \xi_{2} \cos \varphi & J_{O_{2}}
\end{array}\right|
\end{array}\right.
\end{aligned}
$$

\section{Comparison between analytical and numerical solutions}

The form of the system of differential equations 19 reveal that it is unlikely to find an analytical solution and a numerical method should be used in obtaining the where, according to Steiner theorem, [8] there were used the notations:

$$
J_{O_{1,2}}=J_{G_{l, 2}}+M_{1,2} \xi_{1,2}^{2},
$$

for the axial moments of inertia of the rigid bodies with respect to axes normal to the plane of motion, passing through the points $O_{1}$ and $O_{2}$ respectively. For the left member of the equation 1, to the angles $\theta$ and $\varphi$ from relations 3 and 11, the increase $\delta \theta$ and $\delta \varphi$ are considered and then there are calculated the following increases:

$$
\begin{aligned}
\delta \boldsymbol{r}_{G_{l}} & =\boldsymbol{r}_{1}(\theta+\delta \theta)-\boldsymbol{r}_{1}(\theta) \\
\delta \boldsymbol{r}_{G_{2}} & =\boldsymbol{r}_{2}(\theta+\delta \theta, \varphi+\delta \varphi)-\boldsymbol{r}_{1}(\theta, \varphi)
\end{aligned}
$$

Afterwards, the virtual work is calculated using the relation:

$$
\delta L=M_{1} \boldsymbol{g} \delta \boldsymbol{r}_{G 1}+M_{2} \boldsymbol{g} \delta \boldsymbol{r}_{G_{2}}
$$

From relation 17 it is obtained, after the calculus is made:

$$
\delta L=-M_{2} \xi_{2} g \delta \varphi \sin \varphi
$$

The above relations can be employed in establishing the Lagrange equations for the motion of the system, under the following form:

\footnotetext{
* Corresponding author: alaci@fim.usv.ro
}

solution [9]. Given that the system is resolvable with respect to the highest order derivative with respect to the variables $\theta$ and $\varphi$, (relations 20) the Runge-Kutta 4 methodology [10-11] can be applied in solving it. In order to verify the correctness of the equations of motion 20 , a pendulum made of two identical rods, the rod with mobile joint being launched from an angle $\varphi=\pi / 3$ was considered. The pendulum was modelled both with 
the equations 20 and with the software dedicated to dynamical analysis, MSC.ADAMS, as seen in Fig. 3. In Figs. 4 and 5 there are presented comparatively the angular velocities from the two joints of the pendulum, obtained with the simulation software and by integrating the system of differential equations 20 .

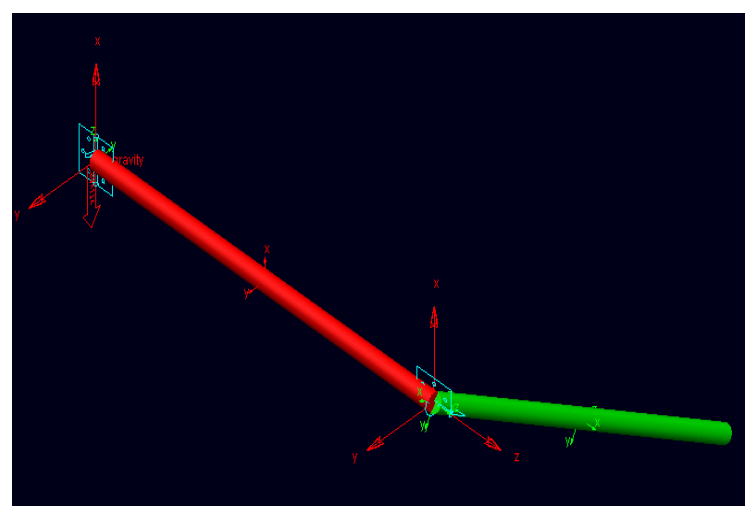

Fig. 3. The pendulum modelled using MSC.ADAMS
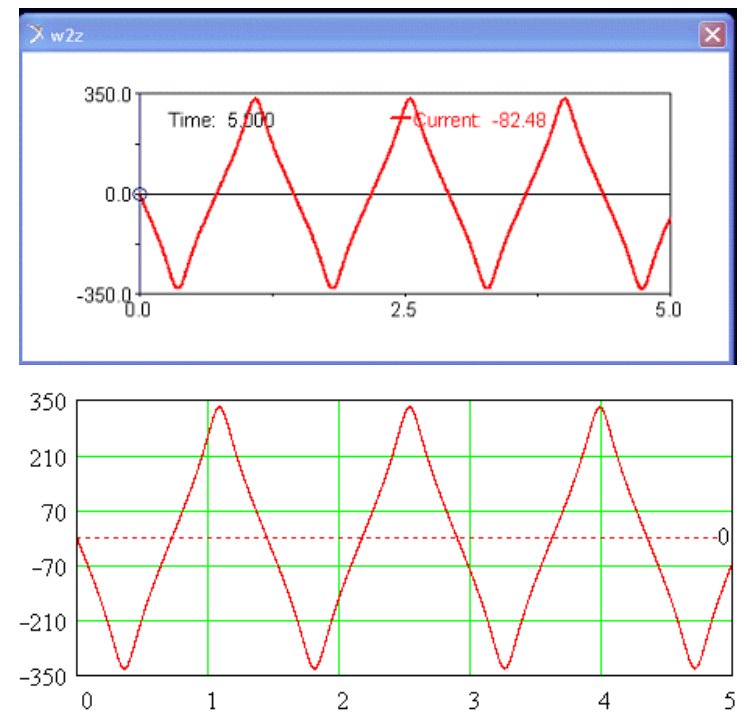

Fig. 4. The angular velocity from the immobile joint of the pendulum
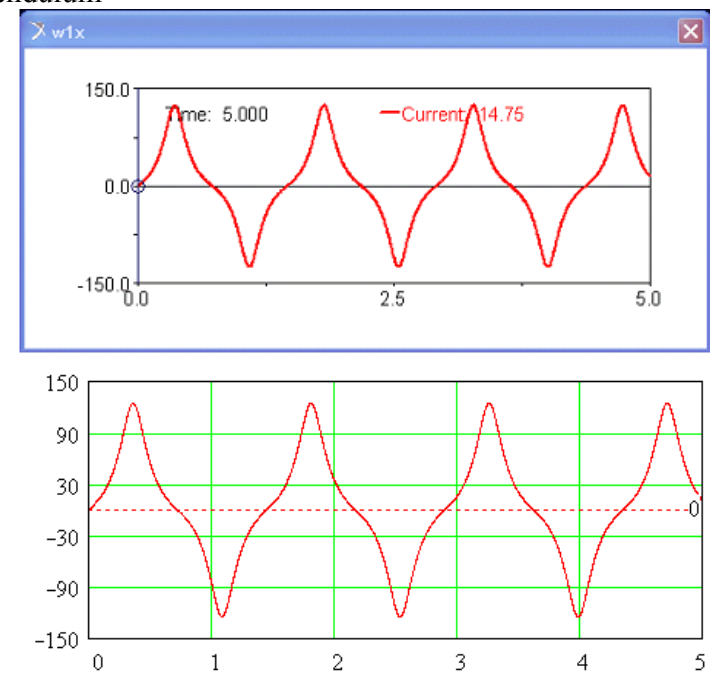

\footnotetext{
* Corresponding author: alaci@fim.usv.ro
}

Fig. 5. The angular velocity from the mobile joint From both plots one can observe the perfect similarity between the results given by the software and the solutions obtained from the equations of motion.

\section{Conclusions}

The paper presents the equations of motion obtained for the spatial pendulum with vertical immobile axis and horizontal mobile axis. The Lagrange's equations of motion were applied for establishing the equations of motion. The equations of motion are obtained for the general situation except for the simplifying assumption that, for each pendulum, the central axial moment of inertia with respect to the axis perpendicular to the axis of oscillations is zero. The nonlinear system of equations was numerically integrated for an actual case. The same pendulum was modelled using dedicated software to dynamical analysis. The perfect concordance between the solutions of the two methods confirms the correctness of the equations of motion obtained in the present work.

This work was supported by a grant of the Romanian Ministry of Research and Innovation, CCCDI - UEFISCDI, project number PN-III-P1-1.2-PCCDI-2017-0404 / 31PCCDI/2018, within PNCDI III.

\section{References}

1. L. Meirovitch, Elements of Vibration Analysis (2nd ed.) (McGraw-Hill Science / Engineering / Math. 1986)

2. H. Alan Lloyd, Some Outstanding Clocks Over 700 Years 1250 - 1950 (Leonard Hill 1958)

3. E. N. Lorenz, J. Atmospheric Sciences, 20 (2) 130141(1963).

4. S. H. Strogatz, Nonlinear Dynamics and Chaos with Applications to Physiscs, Biology, Chemistry and Enginering (CRC Press 2000)

5. D. M. Ardema, Analytical Dynamics: Theory and Applications (Kluwer Academic 2005)

6. J. M. McCarthy, Introduction in Theoretical Kinematics (MIT Press 1990)

7. I. Fischer, Dual-Number Methods in Kinematics, Statics and Dynamics (CRC Press 1995)

8. D. M. Ardema, Newton-Euler Dynamics (2005 Springer)

9. D. W. Jordan, P. Smith, Nonlinear differential equation. Problems and Solutions. A Sourcebook for Scientists and Engineers. Oxford Texts in Applied and Engineering Mathematics (Book 11) (Oxford University Pres 2007)

10. R. Hamming, Numerical Methods for Scientists and Engineers (Dover Publications 1987) 
11. C. W. Gear, Numerical Initial Value Problems in Ordinary Differential Equations (Englewood Cliffs, NJ Prentice-Hall 1971)

* Corresponding author: alaci@fim.usv.ro 